\title{
Rare Occurrence of Bordetella pertussis among Jordanian Children Younger than Two Years Old with Respiratory Tract Infections
}

\author{
Mai G. Jayyosi ${ }^{1}$ Najwa A. Khuri-Bulos ${ }^{2,3}$ Natasha B. Halasa ${ }^{3}$ Samir Faouri ${ }^{4}$ Asem A. Shehabi ${ }^{1}$ \\ ${ }^{1}$ Department of Pathology-Microbiology, The Jordan University, \\ Amman, Jordan \\ 2 Department of Pediatrics, The Jordan University, Amman, Jordan \\ ${ }^{3}$ Department of Pediatrics, Vanderbilt University School of Medicine, \\ Nashville, Tennessee, United States \\ ${ }^{4}$ Department of Pediatrics, Governmental Al Basheer Hospital, \\ Amman, Jordan \\ J Pediatr Infect Dis 2015;10:53-56 \\ Address for correspondence Asem A. Shehabi, DSc, Department of \\ Pathology-Microbiology, Faculty of Medicine, The University of Jordan, \\ Amman, Jordan (e-mail: ashehabi@ju.edu.jo).
}
Abstract
Keywords
- B. pertussis
- M. pneumonia
- C. pneumonia
- L. pneumophila
- Jordanian children

Objectives This study investigated the frequency of the potentially pathogenic bacteria Mycoplasma. pneumoniae, Chlamydia pneumoniae, Legionella pneumophila, Bordetella parapertussis, and Bordetella pertussis among hospitalized Jordanian children. Methods A total of 420 swab samples were collected from the throats and noses of children younger than 2 years. DNA was extracted from the swab samples and was tested for the bacteria being investigated using PCR assays.

Results The results showed the absence of M. pneumoniae, C. pneumoniae, L. pneumophila, and B. parapertussis in all samples. B. pertussis was detected in two samples $(0.48 \%)$ despite DTP vaccine being part of the Jordanian vaccination schedule.

Conclusion This study suggests that certain uncommon bacterial respiratory pathogens rarely cause lower respiratory tract infections in young Jordanian children.

\section{Introduction}

Mycoplasma pneumoniae, Chlamydia pneumoniae, Legionella pneumophila, Bordetella pertussis, and Bordetella parapertussis are important causative agents of respiratory tract infections in children and adults worldwide. ${ }^{1-4}$ The epidemiology of these bacterial agents including their potential contribution to respiratory illness (RI) among Jordanian children is unknown. One study has demonstrated the rare occurrence of M. pneumoniae infection among Jordanian adults with respiratory tract infections. ${ }^{5}$ In addition, since the 1980s almost all Jordanian children have been vaccinated against $B$. pertussis. Cases of classic pertussis have rarely been reported to the Jordanian Ministry of Health over the last two decades. Because of waning immunity, adults may act as reservoirs for infection and transmit infection to unvaccinated or partially vaccinated infants. ${ }^{6}$ B. parapertussis causes classic pertussis-like disease in all age groups, but the disease is milder and of shorter duration.

Microbiological diagnosis of these bacteria is challenging owing to their fastidious nature, and nucleic acid amplification tests such as polymerase chain reaction are currently considered the most useful tests for their laboratory detection. $^{7}$

The aim of this study was to detect M. pneumoniae, C. pneumoniae, L. pneumophila, B. pertussis, and B. parapertussis in a sample of hospitalized Jordanian children admitted with fever and/or respiratory symptoms.

\section{Patients and Methods}

Study design and population. This study is part of a large 3-year prospective study designed to determine the prevalence of common respiratory viral agents in hospitalized Jordanian received

July 24, 2015

accepted after revision

September 16, 2015

published online

November 20, 2015
Copyright $\odot 2015$ by Georg Thieme Verlag KG, Stuttgart · New York
DOI http://dx.doi.org/ 10.1055/s-0035-1567874. ISSN $1305-7707$. 
children younger than 2 years admitted to the main Governmental Al-Basheer Hospital in Amman, Jordan, between 2010 and 2012. The prospective research study was performed by the collaboration of the University of Jordan Hospital, Al-Bashir Hospital, and Vanderbilt University, Tennessee, United States. The study was conducted with the approval of the institutional review boards of Vanderbilt University, University of Jordan, and the Jordanian Ministry of Health. Trained study personnel collected the clinical data from parental interviews and by reviewing medical records.

Sample collection. From each patient, one nasal swab and one throat swab were obtained within 48 hours of admission using sterile cotton swabs. Nasal and throat swabs were mixed together in a 3-mL liquid viral transport media tube specific for viral and chlamydial agents. Each sample was aliquoted into two cryotubes; each cryotube contained $500 \mu \mathrm{L}$ of the sample. The cryotubes were stored at $-80^{\circ} \mathrm{C}$ at the Research Microbiology Laboratory, Faculty of Medicine, University of Jordan. DNA was extracted from thawed specimens using the Qiagen DNA Mini Kit for body fluids (Qiagen, Hiden, Germany) according to the manufacturer's protocols.

Laboratory testing. A multiplex polymerase chain reaction (PCR) was used to detect $M$. pneumoniae, C. pneumoniae, and L. pneumophila in patients' samples as reported by Strålin et al and Jaulhac et al. ${ }^{8,9}$ For every PCR run, a negative control and three positive controls were included. The negative control composed of all the amplification mixture except the purified DNA. Extracted DNA controls of C. pneumoniae, M. pneumoniae, and L. pneumophila were used as positive controls. Another multiplex PCR was designed to detect B. pertussis and B. parapertussis. ${ }^{10}$ To assess the quality and quantity of the extracted DNA from clinical samples, the presence or absence of human DNA was determined using a PCR assay targeting exon-1 of $\beta$-globin gene. ${ }^{11} \mathrm{~A}$ positive test result for $\beta$-globin gene indicates that the sample contained DNA of sufficient quality and quantity for PCR amplification. - Table 1 shows the sequences of the primers and their amplification sizes.

\section{Results}

A total of 420 hospitalized Jordanian children younger than 2 years were enrolled in this study. There were 243 (57.8\%) males, 177 (42.2\%) females, and the mean age was 5.8 months
(-Table 2). The majority of patients were diagnosed with lower respiratory tract infections (70.5\%); 146 (34.7\%) had bronchopneumonia, 111 (26.4\%) had suspected sepsis, 95 (22.6\%) had bronchiolitis, 34 (8\%) had pneumonia, and 21 (5\%) were diagnosed with pertussis or pertussis-like cough. The mean duration of hospitalization was 5 days, ranging from 0 to 26 days, and 157 (37.3\%) patients had received antibiotic treatment prior to hospitalization. The main symptoms were cough $(n=321[76.4 \%])$, fever $(n=242$ [57.6\%]), vomiting (65 [15.4\%]) and diarrhea (40 [9.5\%]). All 420 samples tested negative for C. pneumoniae, M. pneumoniae, L. pneumophila, and $B$. parapertussis, whereas two samples $(0.48 \%)$ were positive for $B$. pertussis. These two cases had not been vaccinated with DTP. Both cases presented with cough and shortness of breath; one case was associated with pneumonia and the second with cyanosis.

\section{Discussion}

In this study, C. pneumoniae, M. pneumoniae, L. pneumophila, and B. parapertussis were not detected; however, two cases of B. pertussis were detected. Given the age group of these children, this was not surprising. Reports of infections with these bacterial agents are usually observed in older children. However, since this study was performed during a 3-year period, it is possible that these agents were not causing sporadic outbreaks of diseases over these years. ${ }^{7,12,13}$ In addition, about one-third of our investigated children (37.3\%) were treated with antibiotics before admission to the hospital, which might have affected the results of the study. Recent studies from African and European countries have reported the prevalence of $M$. pneumoniae infections ranging from 2 to $4.5 \%$ among school-age children and infants younger than 1 year. ${ }^{2,14,15}$ Other studies have also confirmed the low prevalence of $M$. pneumoniae in children younger than 2 years. ${ }^{14,16}$

The present study also revealed that L. pneumophila was not present in this cohort of hospitalized children. This was also not surprising because legionella infection is rarely observed among children and legionella bacteria are mostly associated with hot water in spas and air conditioning systems. ${ }^{6,17}$ In Jordan, central air conditioning systems are not widely used during the whole year, and spas are not available for the majority of population. It is

Table 1 The sequence of primers and their amplification sizes used in the PCR assay for detection of Chlamydia pneumoniae, Mycoplasma pneumoniae, Legionella pneumophila, Bordetella pertussis, and Bordetella parapertussis

\begin{tabular}{|l|l|l|l|l|}
\hline Microorganism & Gene target & Forward primer & Reverse primer & Amplicon size (bp) \\
\hline C. pneumoniae & MOMP & ACACGATGCAGAGTGGTTCA & TGTTTACAGAGAATTGCGATACG & 368 \\
\hline M. pneumoniae & P1 & ACTCGGAGGACAATGGTCAG & CAAACCCGGTCTTTTCGTTA & 483 \\
\hline L. pneumophila & mip & GGTGACTGCGGCTGTTATGG & GGCCAATAGGTCCGCCAACG & 600 \\
\hline B. pertussis & IS481 & GATTCAATAGGTTGTATGCATGGTT & TGGACCATTTCGAGTCGACG & 151 \\
\hline B. pertussis & PTxA & GCGCATGCGTGCAGATTCGTC & CCCTCTGCGTTTTGATGGTGCC & 190 \\
\hline B. parapertussis & IS1001 & CGCCGCTTGATGACCTTGATA & CACCGCCTACGAGTTGGAGAT & 498 \\
\hline
\end{tabular}


Table 2 Demographic characteristics of the examined patients

\begin{tabular}{|c|c|c|}
\hline & $n$ & $\%$ \\
\hline Total number of patients & 420 & 100 \\
\hline Female & 177 & 42.2 \\
\hline Male & 243 & 57.8 \\
\hline Mean age at enrollment (mo) & 5.8 & - \\
\hline Mean duration of hospitalization (d) ${ }^{a}$ & 5 & - \\
\hline Antibiotic treatment prior to hospitalization & 157 & 37.3 \\
\hline No. of patients with cough & 321 & 76.4 \\
\hline No. of patients with fever & 242 & 57.6 \\
\hline No. of patients with vomiting & 65 & 15.4 \\
\hline
\end{tabular}

${ }^{\mathrm{a}}$ Four patients out of 420 were not hospitalized.

also important to note that respiratory therapy devices and shower heads can contain water contaminated with legionella, and hospital-acquired legionella infection has been reported in children who are immunocompromised. ${ }^{18}$ Children with nosocomial infection were not included in this study because samples were collected within 48 hours of hospital admission, and this period is shorter than the incubation period required for infection with $L$. pneumophila (2-10 days) ${ }^{18} \mathrm{~A}$ recent study has reported that infection with $L$. pneumophila, tested for using PCR and urinary antigen test, was absent in hospitalized children between 2 months and 15 years old. ${ }^{18}$

This study detected two children who tested positive for B. pertussis $(0.48 \%)$. The two positive cases were infants younger than 3 months (21 days and 2 months) and had not received their DTP vaccine. Neither of these infants were treated with antibiotics prior to hospitalization. The age group of these children are consistent with other reports of infants hospitalized with pertussis ( $<3$ months of age). ${ }^{19,20}$ Several studies from different regions have reported that the frequency of $B$. pertussis in infants ranged from 5.4 to $16.3 \%{ }^{6,19,20}$ The prevalence of $B$. pertussis varies widely from one study to another according to age group, the number of patients examined, the laboratory method (e.g., PCR, ELISA, culture), and type of samples used to test for B. pertussis, as well as the immunization status of the patients. A surveillance study of pertussis performed in French hospitals found that only $1 \%$ of infants presenting with symptoms of whooping cough over a period of 10 years had B. parapertussis. ${ }^{4}$ Recent studies have reported that the prevalence of $B$. parapertussis in children was 0.25 and $1.3 \%$ in Ireland and Tunisia, respectively. ${ }^{19,20}$

In Jordan, infection with B. pertussis or B. parapertussis can be diagnosed only by clinical examination, as other laboratory assays are not widely available. This fact might underestimate the true prevalence of B. pertussis/B. parapertussis, particularly among infants with mild whooping cough which are occasionally observed in this country. Despite the low rate of positive B. pertussis cases and the absence of $B$. parapertussis cases in this study, it is still important to note that any single positive case among hospitalized infants might result in an outbreak of pertussis infection among children not fully immunized.

Conflict of Interest

The authors declare that there are no conflicts of interest.

\section{Acknowledgments}

The authors are thankful for the financial support (no. 70/2011-2012) by the Scientific Research Deanship of the University of Jordan, Amman, Jordan. All phases of this project were also supported by Union Bank of Switzerland (UBS) Optimus Foundation. The project publication described was supported by CTSA award no. UL1TR000445 from the National Center for Advancing Translational Sciences. Its contents are solely the responsibility of the authors and do not necessarily represent official views of the National Center for Advancing Translational Sciences or the National Institutes of Health. Also, we wish to acknowledge our research staff who help in enrolling these patients: Hanan Amin, Amani Altaber, Hana'a Khalaf, Isra'a Kharbat, Darin Yasin, and Shireen Issa.

\section{References}

1 Cherry JD, Seaton BL. Patterns of Bordetella parapertussis respiratory illnesses: 2008-2010. Clin Infect Dis 2012;54(4): 534-537

2 Cunha BA. The atypical pneumonias: clinical diagnosis and importance. Clin Microbiol Infect 2006;12(Suppl 3):12-24

3 Touati A, Pereyre S, Bouziri A, et al. Prevalence of Mycoplasma pneumoniae-associated respiratory tract infections in hospitalized children: results of a 4-year prospective study in Tunis. Diagn Microbiol Infect Dis 2010;68(2):103-109

4 Bonmarin I, Levy-Bruhl D, Baron S, et al. Pertussis surveillance in French hospitals results from a 10 year period. Euro Surveill 2007; 12(1):678

5 Obeidat NM, Qatouseh Luay F, Shehabi AA. A rare occurrence of Mycoplasma pneumoniae infection among Jordanian adults with respiratory tract infections. Microb Ecol Health Dis 2005; 17:216-218

6 Tan T, Trindade E, Skowronski D. Epidemiology of pertussis. Pediatr Infect Dis J 2005;24(5, Suppl):S10-S18

7 Wolf J, Daley AJ. Microbiological aspects of bacterial lower respiratory tract illness in children: atypical pathogens. Paediatr Respir Rev 2007;8(3):212-219, quiz 219-220

8 Strålin K, Bäckman A, Holmberg H, Fredlund H, Olcén P. Design of a multiplex PCR for Streptococcus pneumoniae, Haemophilus influenzae, Mycoplasma pneumoniae and Chlamydophila pneumoniae to be used on sputum samples. APMIS 2005; 113(2):99-111

9 Jaulhac B, Nowicki M, Bornstein N, et al. Detection of Legionella spp. in bronchoalveolar lavage fluids by DNA amplification. J Clin Microbiol 1992;30(4):920-924

10 Dragsted DM, Dohn B, Madsen J, Jensen JS. Comparison of culture and PCR for detection of Bordetella pertussis and Bordetella parapertussis under routine laboratory conditions. J Med Microbiol 2004;53(Pt 8):749-754 
11 Krajinovic M, Labuda D, Richer C, Karimi S, Sinnett D. Susceptibility to childhood acute lymphoblastic leukemia: influence of CYP1A1, CYP2D6, GSTM1, and GSTT1 genetic polymorphisms. Blood 1999; 93(5):1496-1501

12 Fisman DN, Lim S, Wellenius GA, et al. It's not the heat, it's the humidity: wet weather increases legionellosis risk in the greater Philadelphia metropolitan area. J Infect Dis 2005;192(12):2066-2073

13 Vervloet LA, Marguet C, Camargos PA. Infection by Mycoplasma pneumoniae and its importance as an etiological agent in childhood community-acquired pneumonias. Braz J Infect Dis 2007; 11(5):507-514

14 Defilippi A, Silvestri M, Tacchella A, et al. Epidemiology and clinical features of Mycoplasma pneumoniae infection in children. Respir Med 2008;102(12):1762-1768

15 Lassmann B, Poetschke M, Ninteretse B, et al. Community-acquired pneumonia in children in Lambarene, Gabon. Am J Trop Med Hyg 2008;79(1):109-114
16 Touati A, Pereyre S, Bouziri A, et al. Prevalence of Mycoplasma pneumoniae-associated respiratory tract infections in hospitalized children: results of a 4-year prospective study in Tunis. Diagn Microbiol Infect Dis 2010;68(2):103-109

17 Palusińska-Szysz M, Cendrowska-Pinkosz M. Pathogenicity of the family Legionellaceae. Arch Immunol Ther Exp (Warsz) 2009;57(4): 279-290

18 Diederen BM. Legionella spp. and Legionnaires' disease. J Infect 2008;56(1):1-12

19 Grogan JA, Logan C, O'Leary J, Rush R, O'Sullivan N. Real-time PCRbased detection of Bordetella pertussis and Bordetella parapertussis in an Irish paediatric population. J Med Microbiol 2011;60(Pt 6):722-729

20 Zouari A, Smaoui H, Brun D, et al. Prevalence of Bordetella pertussis and Bordetella parapertussis infections in Tunisian hospitalized infants: results of a 4-year prospective study. Diagn Microbiol Infect Dis 2012;72(4):303-317 